\title{
Right Atrial Mass in a Patient With COVID-19 Pneumonia: a case report
}

\author{
Fahmi Othman ${ }^{1}$, Abdul Rehman Abid ${ }^{1}$, Sabir Abdulkarim ${ }^{1}$, Mohamad Khatib ${ }^{1}$, \\ Abdulqadir Nashwan ${ }^{1}$, Maryam Alkuwari ${ }^{1}$, Abdulwahid AlMulla ${ }^{1}$, and Awad Alqahtani ${ }^{1}$ \\ ${ }^{1}$ Hamad Medical Corporation
}

March 27, 2021

\begin{abstract}
Lipomatous hypertrophy of the interatrial septum (LHIAS) is a benign cardiac tumor. Differential diagnosis of LHIAS consists of atrial masses such as myxomas or lipomas. Herein, we report a 66-year-old male, admitted as a case of severe COVID-19 and was found to have a LHIAS extending to the crista terminalis.
\end{abstract}

Right Atrial Mass in a Patient With COVID-19 Pneumonia: a case report

Fahmi Othman ${ }^{1}$, Abdul Rehman Abid ${ }^{1}$, Sabir Abdulkarim ${ }^{1}$, Mohamad Y. Khatib ${ }^{2}$, Abdulqadir J. Nashwan ${ }^{4}$, Maryam Alkuwari ${ }^{3}$, Abdulwahid AlMulla ${ }^{1}$, Awad Alqahtani ${ }^{1}$

${ }^{1}$ Department of Cardiology and Cardiovascular Surgery, Heart Hospital, Hamad Medical Corporation, ${ }^{2}$ Critical Care and Pulmonary Medicine, Hamad Medical Corporation, ${ }^{3}$ Department of Radiology, Hamad Medical Corporation, ${ }^{4}$ Departement of Nursing Education and Research, Hamad Medical Corporation, Doha, Qatar.

Corresponding author:

Mr. Abdulqadir J. Nashwan

anashwan@hamad.qa

Tel: $(+974) 40240487$

Mob: (+974) 66473549

P.O.Box 3050 Doha, Qatar

\section{ABSTRACT}

Lipomatous hypertrophy of the interatrial septum (LHIAS) is a benign cardiac tumor. Differential diagnosis of LHIAS consists of atrial masses such as myxomas or lipomas. Herein, we report a 66-year-old male, admitted as a case of severe COVID-19 and was found to have a LHIAS extending to the crista terminalis.

Keywords: Lipomatous hypertrophy; interatrial septal hypertrophy; COVID-19; Atrial mass; benign cardiac tumor.

\section{Key Clinical Message:}

Lipomatous hypertrophy of the interatrial septum can have an atypical appearance by transthoracic echocardiography. The authors emphasize on the importance of the multimodality imaging approach to reach the appropriate diagnosis in such cases. 


\section{BACKGROUND}

Lipomatous hypertrophy of the interatrial septum (LHIAS) is a well-described benign cardiac tumor. LHIAS is a quite frequent finding seen by echocardiography. It is estimated to be presented in up to $8 \%$ of the general population. (1)

LHIAS is typically diagnosed by Transthoracic echocardiography (TTE). However, in some cases, it may have an atypical appearance by TTE, especially when the echo images are suboptimal.

Differential diagnosis consists of atrial masses such as myxomas or lipomas.(2)

Interatrial septum thickness of $6 \mathrm{~mm}$ in the general population and $7 \mathrm{~mm}$ in the elderly are considered normal.(3) Lipomatosis has been defined as a thickness greater than $15 \mathrm{~mm}$.(4) Adipose tissue can also accumulate in sub epicardium, crista terminalis, endocardium, and the mediastinum.

\section{CASE PRESENTATION}

This is a case of a 66-year-old man with a previous history of hypertension, diabetes, chronic kidney disease, and psoriasis. He presented to the communicable disease center (CDC) with a three-day history of fever, cough, and shortness of breath. On admission, his vital signs were as follows: blood pressure: $115 / 52 \mathrm{mmHg}$, heart rate: $75 \mathrm{~b} / \mathrm{min}$, respiratory rate: $20 \mathrm{~b} / \mathrm{min}$, and oxygen saturation $\left(\mathrm{O}_{2}\right.$ sat $): 93 \%$ on room air. His body mass index (BMI) was $29 \mathrm{~kg} / \mathrm{m}^{2}$. Physical examination was remarkable for bilateral chest crackles with normal cardiovascular examination.

His electrocardiogram (ECG) revealed a sinus rhythm with right bundle branch block (RBBB) and left axis deviation. Chest x-ray showed patchy peripheral ground-glass opacities in both lungs and soft consolidations in the right middle lobe. Polymerase chain reaction (PCR) was performed on a nasopharyngeal swab and returned positive for SARS-CoV-2. In addition, blood tests showed WBC of $5.9 \times 10^{\wedge} 3 / \mathrm{uL}$, hemoglobin of $10 \mathrm{gm} / \mathrm{dL}(\mathrm{N}\{13-17 \mathrm{gm} / \mathrm{dL}\})$, C-reactive protein (CRP) of $146 \mathrm{mg} / \mathrm{L}[\mathrm{N}<6 \mathrm{mg} / \mathrm{L}])$, ferritin of $2619 \mathrm{ug} / \mathrm{L}$ $[\mathrm{N}<553 \mathrm{ug} / \mathrm{L}])$, creatinine of $190 \mathrm{umol} / \mathrm{L}(\mathrm{N}\{62-106 \mathrm{umol} / \mathrm{L})$, and D Dimer of $2.3 \mathrm{mg} / \mathrm{L}$ FEU $[\mathrm{N}<0.5$ $\mathrm{mg} / \mathrm{L}$ FEU]).

He was started on oxygen therapy at 2 liters per minute via nasal cannula, but shortly after admission, he became severely hypoxic, and then, he was transferred to the medical intensive care unit (MICU) and kept on continuous positive airway pressure (CPAP) to maintain oxygen saturation of $95 \%$.

Transthoracic echocardiography (TTE) was performed, which revealed normal biventricular size and function with no significant valvular disease. Mild pulmonary hypertension with an estimated systolic pulmonary artery pressure of $48 \mathrm{mmHg}$ was noted. The study showed echo dense mass located on the right atrial (RA) roof, best seen in four-chamber view. There was another echo-lucent mass in the RA and protruding into the tricuspid valve annulus, best seen in the RV inflow view, as shown in (Figure 1). A prior TTE report that had been performed in another center one month ago revealed no interatrial mass.

Because of acute COVID-19 pneumonia and elevated D-dimer, the RA mass was considered and treated as a thrombus. He was started on a therapeutic dose of intravenous heparin and warfarin; thus, the INR was maintained in the therapeutic range between 2 to 3 . Repeated echo did not show any significant differences. For a better assessment of the RA mass, Transesophageal echocardiography (TEE) was planned, but was not performed due to acute respiratory distress with low oxygen saturation. Instead, 12 days later, upon clinical recovery a cardiac magnetic resonance imaging (CMR) was performed.

The images were obtained on a 1.5 Tesla scanner (Philips Ingenia), and findings from the multiple stacks of cine four-chamber views confirmed the presence of a structure on the posterior wall of the RA that extends onto the thickened interatrial septum (Figure 2). This smooth structure moved during systole and diastole and was identified as crista terminalis, which was rather prominent. T1 and T2 weighted images on the axial plane of this structure had a similar signal intensity to the subcutaneous fat revealing it as predominantly fatty deposition. T1 weighted images with fat saturation sequences (Figure 3) demonstrated the suppression of fatty signals consistent with LHIAS of the thickened interatrial septum associated with fatty deposition 
in the crista terminalis. On post gadolinium, early and late acquisition, there were no uptake or features to suggest the presence of thrombus or any other cardiac tumors (Figure 4).

CMR concluded that the prominent RA mass was lipomatosis hypertrophy of the interatrial septum, which extends to the crista terminalis with no evidence of any thrombus. Hence the anticoagulation was discontinued, and patient was discharged home in very good condition.

\section{DISCUSSION}

Lipomatous hypertrophy of the interatrial septum (LHIAS) is usually incidental and typically diagnosed by a transthoracic echocardiogram (TTE) by appreciating the classic dumbbell-shaped morphology within the interatrial septum.(5) However, an atypical appearance on TTE might happen; therefore, multimodality imaging is required to confirm the diagnosis of LHIAS.

LHIAS typically spares the fossa ovalis while respecting the atrial septum boundaries.(6) TTE has low sensitivity in diagnosing LHIAS due to limited resolution, especially when the image quality is suboptimal. A transesophageal echocardiogram can be used to easily make the diagnosis for LHIAS, which best appears 'in the bicaval view' as "globular thickening of the interatrial septum".

What made matters worse was that this mass was incidentally discovered in this patient with COVID-19 pneumonia. As has been published in several studies, accelerated thrombosis was frequently reported in patients admitted with COVID-19. Therefore, in our case, due to severe Covid-19 symptoms with elevated D-dimer, the mass was treated as an intracardiac thrombus; initially, heparin and warfarin, then subsequently with warfarin only using a target INR between 2-3.

Due to the patient's acute respiratory distress, he was not suitable for any intervention. Therefore, the decision was made not to proceed with TEE to prevent further respiratory deterioration.

Typically, the borders of cardiac tumors are determined by CMR. It also offers a superior tissue characterization. LHIAS follow the same signal intensity of subcutaneous fat (7). Sparing the fossa ovalis and extension of greater than $2 \mathrm{~cm}$ in transverse diameter are unique to LHIAS that differentiates it from lipomas. Typical findings include uniform high signal intensity on T1 and T2 weighted images with signal suppression on fatsaturated images.(8) They appear hyperintense also on SSFP cine sequences, with no gadolinium contrast enhancement neither on the first-pass perfusion nor in the delayed phase.(9) Other differential here with fatty content would be malignant masses, which demonstrates infiltration of anatomic structures, poor definition of borders, inhomogeneous tissue appearance post gadolinium, and associated pericardial or pleural effusion.(10) Thus localization, morphology, and signal intensity tissue characterization on the CMR helps differentiate between etiologies of tumors in the heart.

\section{CONCLUSION}

This case highlights the importance of understanding RA anatomy, as well as the limitations of TTE, especially in patients with suboptimal image quality. It has also confirmed the importance of the multimodality imaging approach to reach the appropriate diagnosis in such cases.

\section{Abbreviations}

COVID-19: Coronavirus disease

PCR: Polymerase chain reaction

CMR: Cardiac Magnetic Resonance Imaging

LHIAS: Lipomatous hypertrophy of the interatrial septum

TTE: Transthoracic echocardiography

CPAP: Continuous Positive Airway Pressure

\section{Declarations}




\section{Ethics approval and consent to participate}

The article describes a case report. Therefore, no additional permission from our Ethics Committee was required.

\section{Consent for publication}

The consent for publication was obtained.

\section{Availability of data and material}

All data generated or analyzed during this study are included in this published article.

\section{Competing interests}

The authors declare that they have no competing interests.

\section{Funding}

This study was not funded.

\section{Authors' contributions}

FO, ARA, SA, MYK, AJN, MA, AAM, AAQ: Data Collection, Literature Search, Manuscript Preparation

All authors read and approved the final manuscript

\section{Acknowledgments}

Open Access funding provided by the Qatar National Library.

\section{REFERENCES}

1. Xanthos T, Giannakopoulos N, Papadimitriou L. Lipomatous hypertrophy of the interatrial septum: a pathological and clinical approach. International journal of cardiology. 2007;121(1):4-8.

2. Kılıçkap G. A case with a giant interatrial septal lipomatous hypertrophy, and thickened epicardial and visceral fat: Different faces of a common metabolic problem? Anatolian Journal of cardiology. 2018;19(6):417.

3. Rigatelli G, Dell'Avvocata F, Braggion G, Giordan M, Chinaglia M, Cardaioli P. Persistent venous valves correlate with increased shunt and multiple preceding cryptogenic embolic events in patients with patent foramen ovale: an intracardiac echocardiographic study. Catheterization and Cardiovascular Interventions. 2008;72(7):973-6.

4. Burke AP, Litovsky S, Virmani R. Lipomatous hypertrophy of the atrial septum presenting as a right atrial mass. The American journal of surgical pathology. 1996;20(6):678-85.

5. Kleiman AM, Harding LM, Bechtel AJ. Concomitant lipomatous hypertrophy and left atrial mass: distinguishing benign from malignant. Echocardiography. 2018;35(4):534-6.

6. Gopal DJ, Han Y, Silvestry FE, Ferrari VA. Atypical presentation of lipomatous hypertrophy of the interatrial septum: a case report. European Heart Journal: Case Reports. 2019;3(4):1.

7. Sparrow PJ, Kurian JB, Jones TR, Sivananthan MU. MR imaging of cardiac tumors. Radiographics. 2005;25(5):1255-76.

8. Motwani M, Kidambi A, Herzog BA, Uddin A, Greenwood JP, Plein S. MR imaging of cardiac tumors and masses: a review of methods and clinical applications. Radiology. 2013;268(1):26-43.

9. Dong E. Hongru Du, and Lauren Gardner. 2020.". An Interactive Web-Based Dashboard to Track COVID19 in Real Time.30120-1.

10. Esposito A, De Cobelli F, Ironi G, Marra P, Canu T, Mellone R, et al. CMR in the assessment of cardiac masses: primary malignant tumors. JACC: Cardiovascular Imaging. 2014;7(10):1057-61. 


\section{Hosted file}

Figure 1.pdf available at https://authorea.com/users/346664/articles/515516-right-atrialmass-in-a-patient-with-covid-19-pneumonia-a-case-report

\section{Hosted file}

Figure 2.pdf available at https://authorea.com/users/346664/articles/515516-right-atrialmass-in-a-patient-with-covid-19-pneumonia-a-case-report

\section{Hosted file}

Figure 3.pdf available at https://authorea.com/users/346664/articles/515516-right-atrialmass-in-a-patient-with-covid-19-pneumonia-a-case-report

\section{Hosted file}

Figure 4.pdf available at https://authorea.com/users/346664/articles/515516-right-atrialmass-in-a-patient-with-covid-19-pneumonia-a-case-report 